\title{
Information-Based Construction of High-Speed Railway Tunnel
}

\author{
Qian Yang and Zhaoling Wang \\ Sichuan Agricultural University, Chengdu, Sichuan 611830, China \\ Correspondence should be addressed to Zhaoling Wang; wong8010@berkeley.edu
}

Received 30 June 2017; Accepted 3 October 2017; Published 31 October 2017

Academic Editor: Rafał Burdzik

Copyright (C) 2017 Qian Yang and Zhaoling Wang. This is an open access article distributed under the Creative Commons Attribution License, which permits unrestricted use, distribution, and reproduction in any medium, provided the original work is properly cited.

\begin{abstract}
Nowadays, railway tunnel construction faces huge developments and opportunities, with a tendency for high speed and long distance. How to effectively apply the information in the construction process has been the focus of current research. According to the Xian-nvyan tunnel in Xicheng high-speed railway, our research was based on the geological forecast, selecting appropriate tunneling model parameters to establish the $3 \mathrm{D}$ calculation model. Through the numerical simulation of three tunnel excavation and support methods, we analyzed the displacement of surrounding rock and the plastic failure to select the construction method reasonably. Compared with the actual measured data, we judged the rationality of the selected scheme and model parameters, so as to provide design parameters which conform to the surrounding rock properties for the subsequent construction, thus optimizing the construction program and applying the concept of information-based construction in engineering actually.
\end{abstract}

\section{Introduction}

At present, China has become the largest and the fastestgrowing constructor of tunnels in the world. In midwest traffic construction, tunnel engineering has a great and an unprecedented development opportunity. The characteristics of tunnels include the large scale, complicated technology, wide impact, and high risk, which pose great challenges on the construction of tunnel projects. At the same time, the investigation, design, and construction process of tunnels are in a separating state to a certain degree, resulting in the construction of information that cannot be accessed, transferred, and reacted to timely, thus increasing the construction risk and costs. The insufficient information level of the survey, forecast information, and monitoring measurement data lead to various construction information that cannot be communicated, analyzed, and given feedback on timely and efficiently. Therefore, the comprehensive and efficient integration and the instant feedback of tunnel information have great importance in the high efficiency, safety, and economy of tunnel constructions, which possess different connotations and extensions in different development times.

Since the Austrian civil engineer Rabcewice put forward the new Austrian tunneling method (NATM) in the 1940s, the tunnel construction technology, which proposes to make the most out of the self-bearing capacity of surrounding rock and integrated design, construction, and monitoring, has been a widely used theory of tunnel monitoring measurement. Meanwhile, the method of combining numerical simulation with measurement was also widely applied in informationbased construction. The highly intelligent TMS (Tunnel Measurement System) was used in the Utley tunnel in Switzerland to maximize the measuring time, and it greatly improved the production efficiency and reduced costs [1]. Li Yongsuo's thesis, discussing the numerical simulation for the excavation in tunnel construction, utilized FLAC3D to simulate the mechanical laws of surrounding rock and supporting structure of tunnels and then performed a comparison with the field measurement to optimize the construction scheme and supporting measures [2]. Chen et al. studied the doublearched highway tunnel in Hunan Province, which was based on the field measurements, analyzing the deformation of surrounding rocks and simulating the process of construction with the finite element method [3].

Tunnel engineering depends heavily on the control of construction information; therefore, it is imperative to improve the tunnel information construction. This paper relies on Xicheng Railway Xian-nvyan tunnel, which is a 
high-speed railway tunnel, for the analysis of geological investigation, forecast, numerical simulation, and monitoring measurement, discussing the position and function of the different information in the tunnel construction, in order to provide a reference for the development of tunnel information construction systems.

\section{Project Overview}

Located in Guangyuan City, Sichuan Province, Xian-nvyan tunnel is a sole hole double-line tunnel, with full length of $5633.4 \mathrm{~m}$, maximum depth of $390 \mathrm{~m}$, shallow depth of only $15 \mathrm{~m}$, single hole double-line tunnel spacing of $4.6 \mathrm{~m}$, and ground elevation of 500 970 m.

The surface layer of the study section is mainly strongand weak-weathered sandstone, and the inside part is broken with a gravelly structure. Within the joint fissure development, some areas form perilous rocks, and the diameter of collapsed large stones is several to ten meters. The geological structure is a monocline structure, and the strata tend towards the left line, causing a bedding lateral pressure in the right side of the tunnel, so it is prone to landslide and drop rockfall. Moreover, the groundwater in the tunnel is mainly composed of bedrock fissure water.

\section{Advanced Forecast Information}

Based on the New Austrian Tunneling Method (NATM), tunnel information construction cycles the processes of geological investigation, predesign, simulated construction, and optimization. The information obtained by geological exploration is relatively extensive, which cannot completely control the construction risk and guide the construction, so advanced forecasting becomes an important way to obtain detailed construction information [4].

3.1. Advanced Horizontal Drilling. According to the pregeological investigation, as the mudstone is relatively an aquiclude, the underground part of the sandstone layer forms confined water; thus, multiple boreholes emit groundwater. The MD-50 type DTH (down-the-hole) impact-rotary rig is adopted to drill in the DK425 + 981 tunnel face (which means the tunnel face is 425 kilometers and 981 meters from the railway entrance), with a cumulative drilling length of $23.10 \mathrm{~m}$.

3.2. TSP Geological Forecasting. The normal TSP (Tunnel Seismic Prediction) method mainly consists of the threecomponent geophone, recording cell, and initiation device. The three-component geophone is used to receive seismic wave signals; the recording cell amplifies the received seismic wave signal and performs analog-to-digital conversion and data recording, while also controlling the measuring process, and the initiation device is used for triggering the electric detonator and detonating the explosives and exciting the seismic wave [5].

In this section, parameters such as S-wave velocity, Pwave-to-S-wave velocity ratio, and Poisson's ratio change greatly, with fracture water developing, indicating that the rock condition is worse than the previous one, especially the representative segment, DK425 + 968 DK425 + 980, owning concentrated reflecting layers. Based on the above analysis, it is inferred that the fault fracture zone existed, suggesting that the section should be constructed by grade IV surrounding rock, noting the partial collapse and piece dropping of the construction, paying attention to the support treatment and construction safety.

On the basis of the pregeological investigation, through the rock mechanics parameters of the geological forecast, shown in Figure 1, and groundwater and karst conditions in front of the tunnel face, we can forecast the width, occurrence, and location of the forward fault zone. In addition, we also obtain the geological investigation and provide the parameter guarantee for the establishment of a numerical simulation model [6].

\section{Numerical Simulation}

The numerical method of tunnel engineering is an effective method to solve the tunnel engineering problem, which heavily depends on the research of the engineering geological conditions and rock property, reflected in the simulation results through the parameters. In this paper, three main tunnel excavation methods are simulated by FLAC3D; the optimal excavation method can be chosen, making a reasonable evaluation and prediction of the stability of tunnel surrounding rock, so as to direct the follow-up construction [7].

4.1. Numerical Model. The numerical test parameters are adopted from the "Highway Tunnel Design Code" (JTG D702004), combined with the advanced geological forecast. Figure 2 shows a longitudinal section drawing of the simulation section in order to prove the geological condition in front of the tunnel face before construction, and the advanced geological drilling and TSP forecasting data indicate that the tunnel rock of the test section was magenta and highly weathered sandstone, which is loose and soft, explaining the integrity and poor self-stability [8]. As a result of the test section tunnel's weak water, the calculation parameters of surrounding rock are reduced to a certain extent [9].

Based on the TSP forecasting and the geological condition, we calculate the parameters of the tunnel model shown in Table 1, which provides accurate information for model building. The real tested tunnel is a sole hole double-line one with a span of $13.92 \mathrm{~m}$. As shown in Figure 3, the dimensions of the calculated model are $112 \mathrm{~m}$ width, eight times the span, $92 \mathrm{~m}$ height, and $52 \mathrm{~m}$ longitudinal dimension. For the shallow tunnel depth, in order to simulate the actual situation of the test section, the depth of the model is $15 \mathrm{~m}$, and the surface unsymmetrical pressure angle is 10 degrees.

In view of the good stability of the surrounding rock and no obvious rheological behavior after excavation, the geological condition is relatively stable. Therefore, the numerical model can be calculated according to the elastoplastic theory, and the Mohr-Coulomb criterion is adopted to describe the mechanical behavior of rock mass, which contains the shear failure criterion and tensile failure criterion. 


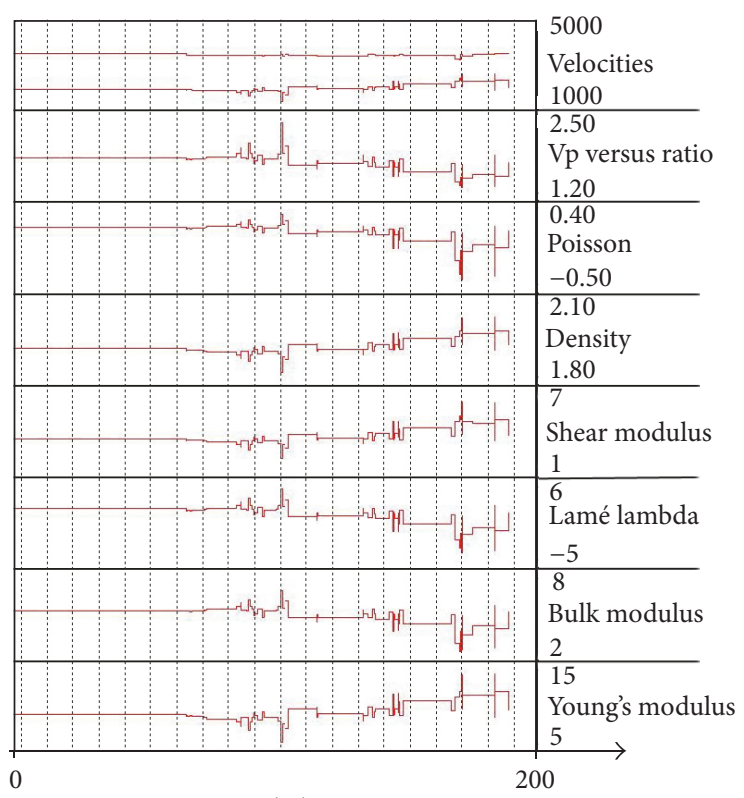

(m)

FIGURE 1: Rock mechanics parameters of the geological forecast.

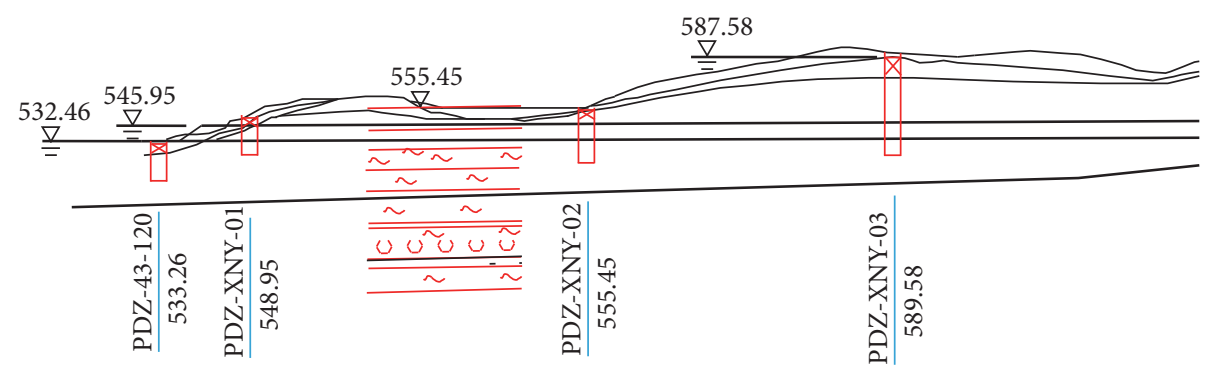

FIGURE 2: Longitudinal section drawing of the simulation section.

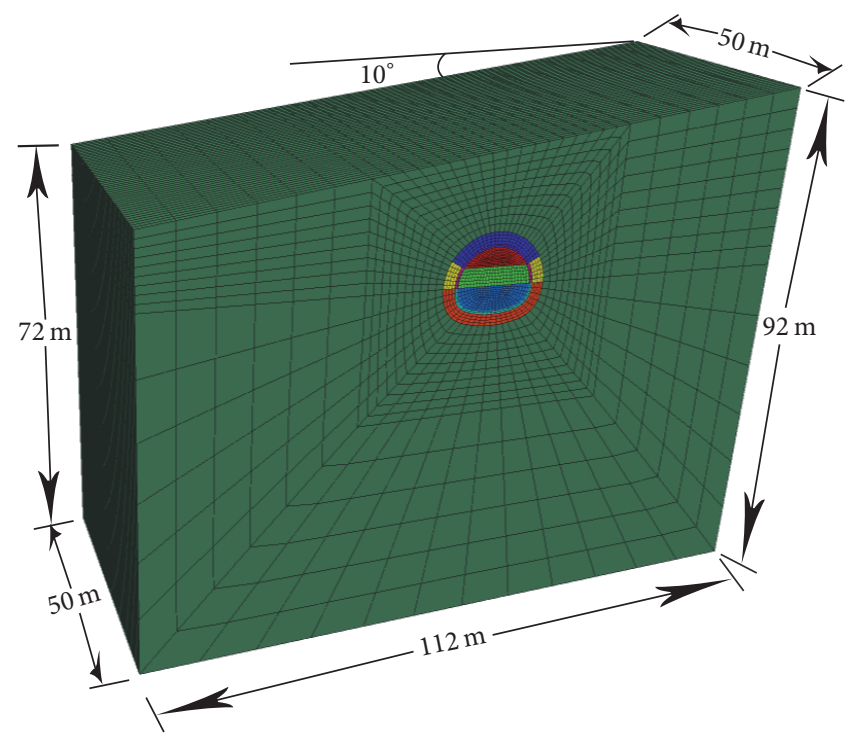

Figure 3: Numerical model. 
TABLE 1: Calculation parameters of the tunnel model.

\begin{tabular}{|c|c|c|c|c|c|c|}
\hline Number & Material & $\begin{array}{c}\text { Elastic modulus } \\
(\mathrm{GPa})\end{array}$ & Poisson's ratio & $\begin{array}{c}\text { Cohesion } \\
(\mathrm{MPa})\end{array}$ & $\begin{array}{c}\text { Internal friction } \\
\left({ }^{\circ}\right)\end{array}$ & $\begin{array}{c}\text { Bulk density } \\
\left(\mathrm{kN} / \mathrm{m}^{3}\right)\end{array}$ \\
\hline (1) & Surrounding rock & 1.5 & 0.35 & 0.1 & 22 & 19.6 \\
\hline (2) & Primary support & 28 & 0.2 & - & - & 24.5 \\
\hline (3) & Grouting in surrounding rock & 6 & 0.35 & 0.7 & 39 & 24.5 \\
\hline (4) & Secondary lining & 30 & 0.2 & - & - & 24.5 \\
\hline
\end{tabular}

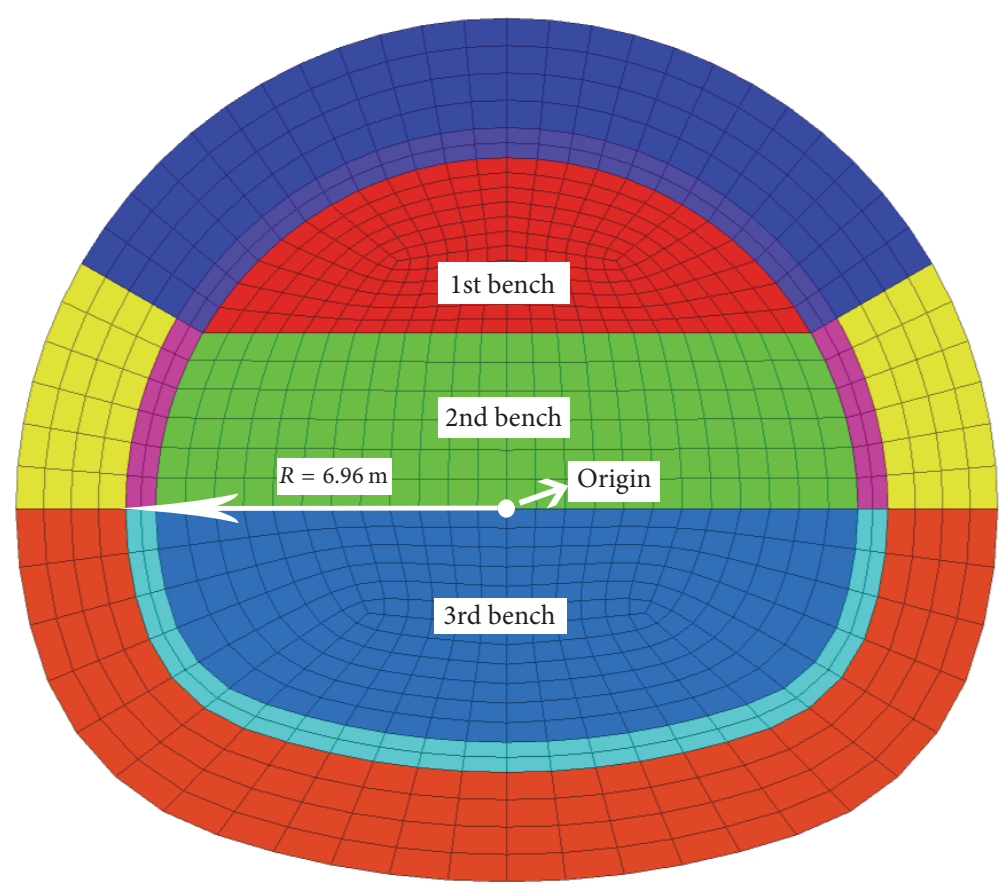

FIgURE 4: Numerical model of the excavation.

The boundary condition for the model is fixed in all directions on the bottom side, normal direction on the side boundaries. The sign for the horizontal displacement is negative (deform to the left) or positive (deform to the right). The sign for the vertical displacement is negative (deform downwards) or positive (deform upwards).

This experiment simulates three excavation methods in Figure 4, that is, full section, two-bench section, and tribench section, monitoring the clearance convergence, ground settlement, and the plastic zone failure of the midpoint section of the model. By comparing the deformation law rule of surrounding rock in different construction methods, analyzing the rock mechanical behavior, and choosing more suitable schemes for this project in excavation and support, the role of numerical simulation technology in tunnel information model is illustrated as an example [10].

\subsection{The Result of the Numerical Experiment}

4.2.1. Analysis of Clearance Convergence. In order to compare the developing process of clearance convergence in different conditions, the paper has taken the mid cross section of the 50-meter tunnel as the monitoring section. Every two meters of the excavation footage tunneling, we extract the deformation data of the mid cross section. The beginning of the monitoring is when the up-bench was dug to $15 \mathrm{~m}$, and it ends as the tunnel was dug through.

The results in Figure 5 depict the clearance convergence change of the selected section in the process of tunnel excavation. The displacement of all nodes around the hole is magnified 18 times to make the described graph more intuitive.

It can be seen from Figure 5 that the evolution of the tunnel convergence deformation after excavation is closely related to the construction method. In virtue of the unsymmetrical pressure, leading to the skew of the tunnel section, the tunnel convergence deformation tends towards the deeply buried direction.

By comparing the deformation results, we found that the convergence deformation of the full section is relatively small and uniform, which is influenced by the excavation of adjacent sections, and there is no large horizontal or vertical displacement; hence, the deformation rate gradually decreased and then stabilized. 


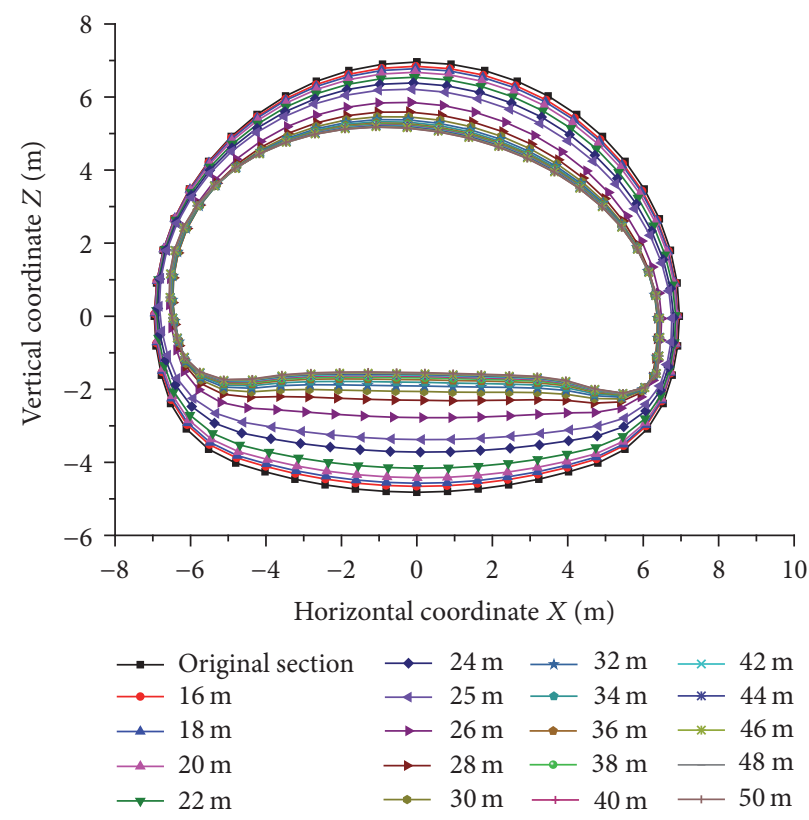

(a) Full section

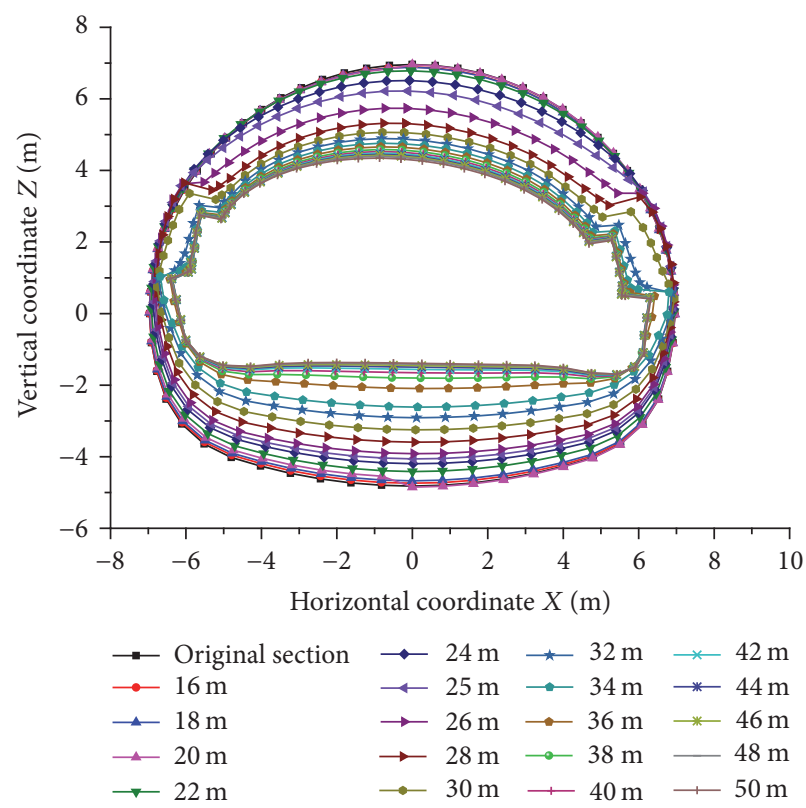

(c) Tribench

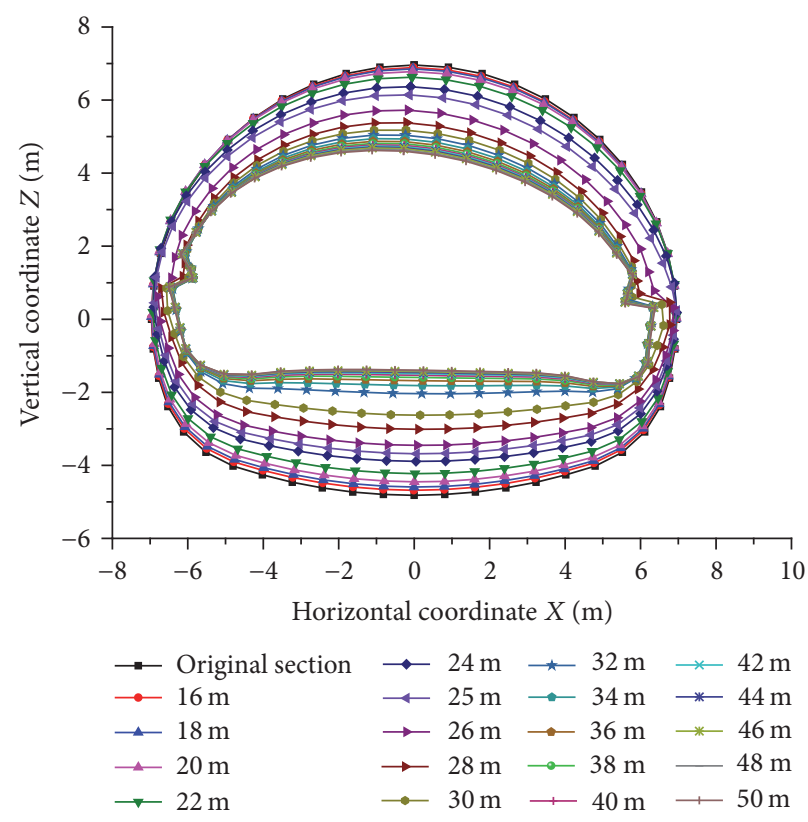

(b) Two-bench section

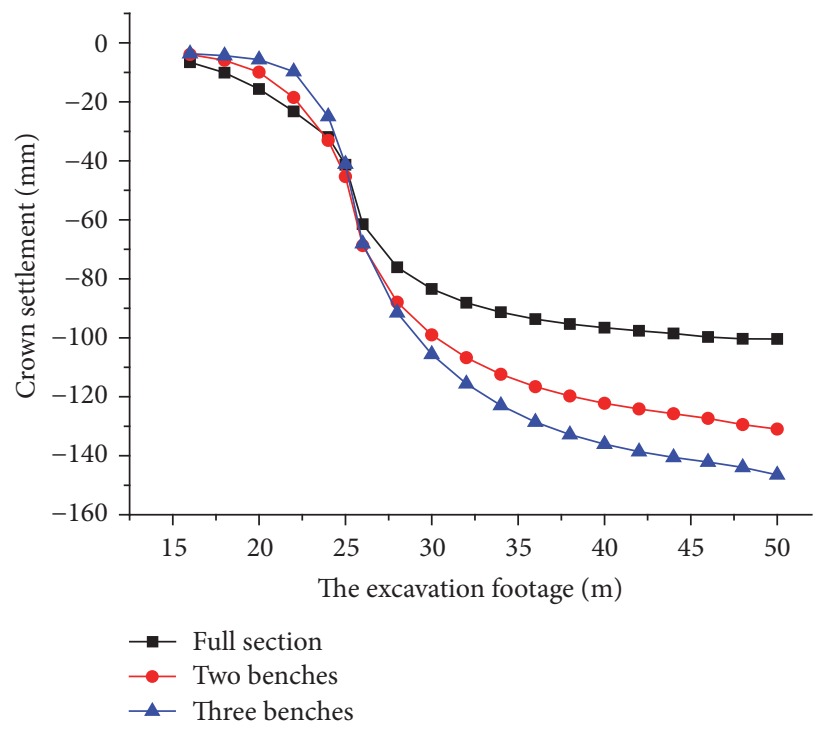

(d) Crown settlement of the central line

FIGURE 5: The clearance convergence by different methods.

However, in the step excavation, due to the excavation and support disturbance of previous processes and the uppermiddle steps, the convergence displacement is not uniform after the excavation of the steps in the monitoring section, and each step, especially the arch, will cause a great deformation and then tend to stabilize. Among these, the vertical deformation of the tribench method is larger than the two-bench method, so the advance support should be strengthened and the surrounding rock ought to be closed into a ring timely in the construction.
In addition, the paper also took the crown settlement of the central line into consideration. From the curve, the deformation of the full-section excavation is minimum among the three methods, which can stabilize in a relatively short time, which requires that the support must be in place timely, increasing the difficulty and insecurity of the support. At the same time, the tribench excavation has the greatest influence on the crown settlement, followed by the two-bench method, and the cumulative deformation is 1.7 and 1.4 times, respectively, the full-section excavation. 


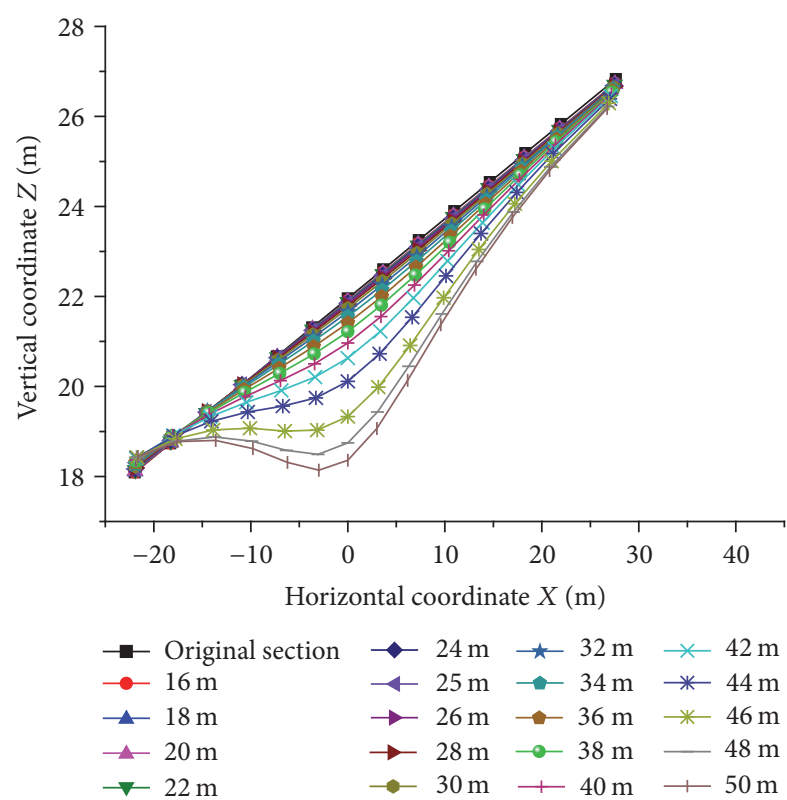

(a) Full section

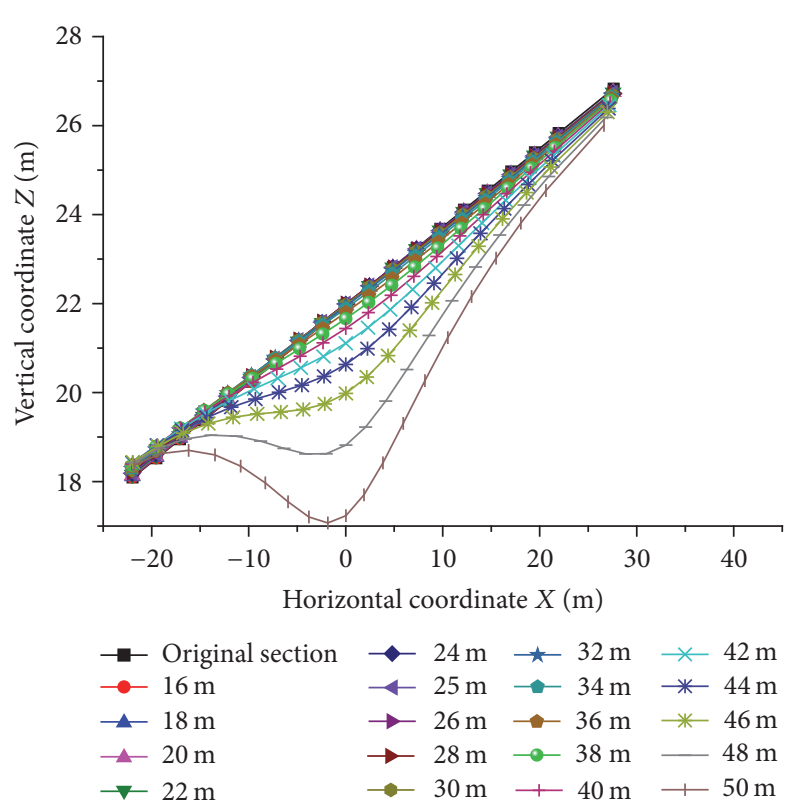

(c) Tribench

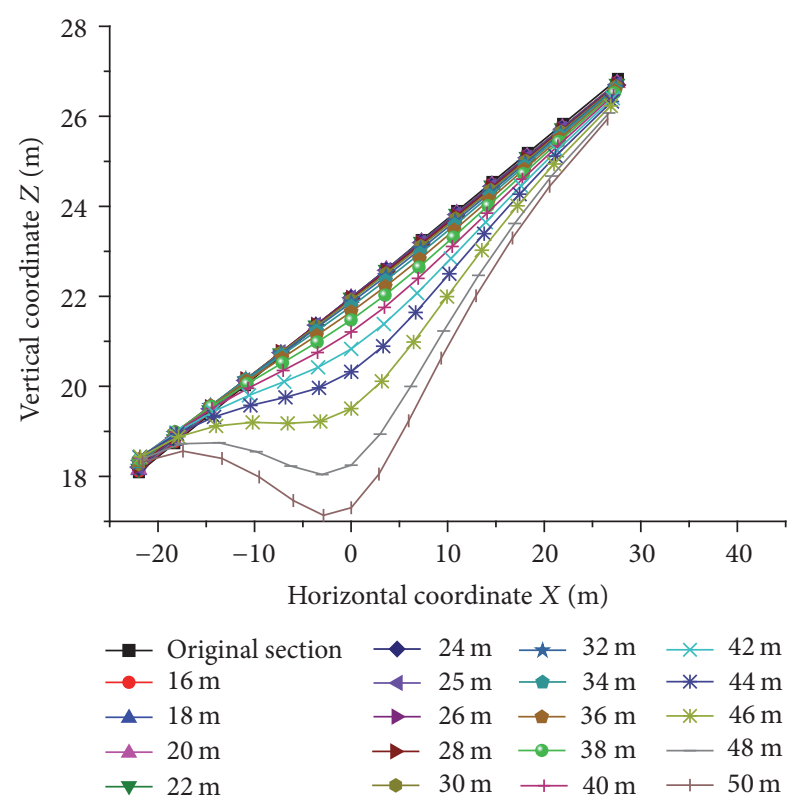

(b) Two-bench section

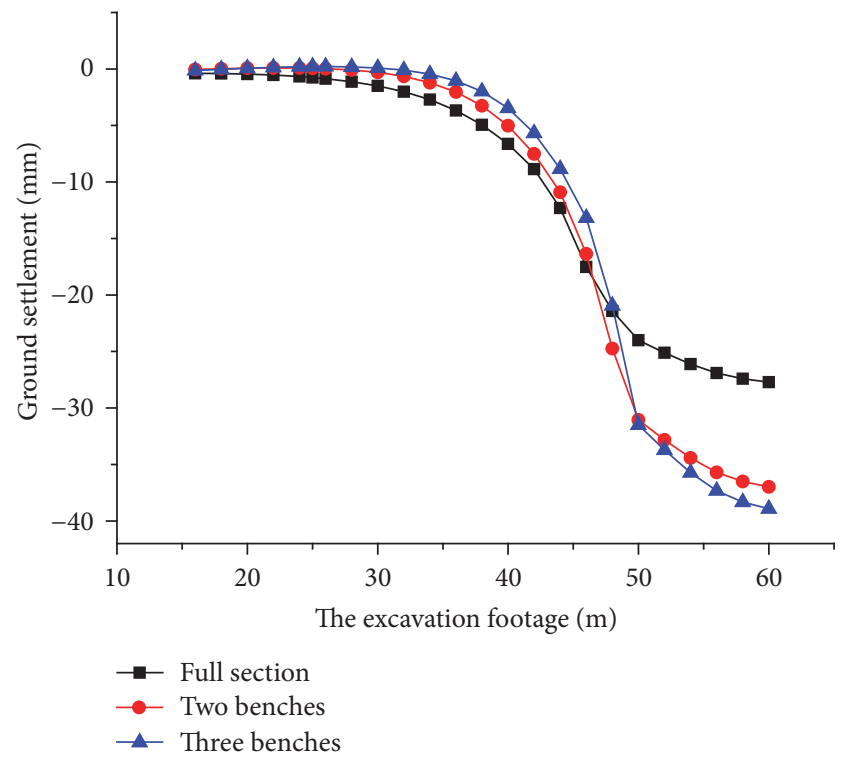

(d) Crown settlement of the central line

FIgURE 6: The ground settlement by different methods.

4.2.2. Analysis of Ground Settlement. The observation of ground settlement is mainly to investigate the influence of different construction methods. Same as the clearance convergence, the paper monitored the displacement of the mid cross section and took a record every 2 meters forward. In the simulated calculation, seven monitoring points have been placed in the selected cross section, with the ground settlement value expanding 150 times to superimpose directly on the curve that shows the surface node location.

The results are plotted as a sectional groove curve, as Figure 6 shows.
From the ground displacement monitoring data, the maximum sedimentation value is distributed in the vicinity of the centerline of the tunnel section.

As shown in Figure 6, the three kinds of excavation methods did not cause large ground settlement immediately after the excavation of the monitoring section, and the decreasing rate of settlement which is caused by excavation, with the increase of the distance to the tunnel face, is essentially in agreement.

With the excavation, the ground settlement began to increase significantly in the $38 \mathrm{~m}$ footage, decreasing 


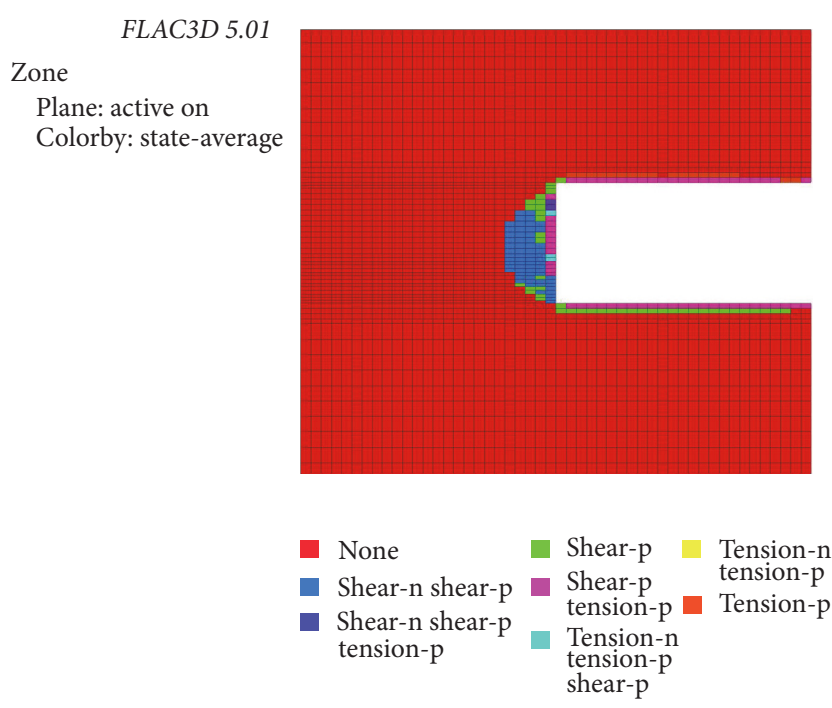

(a) Full section

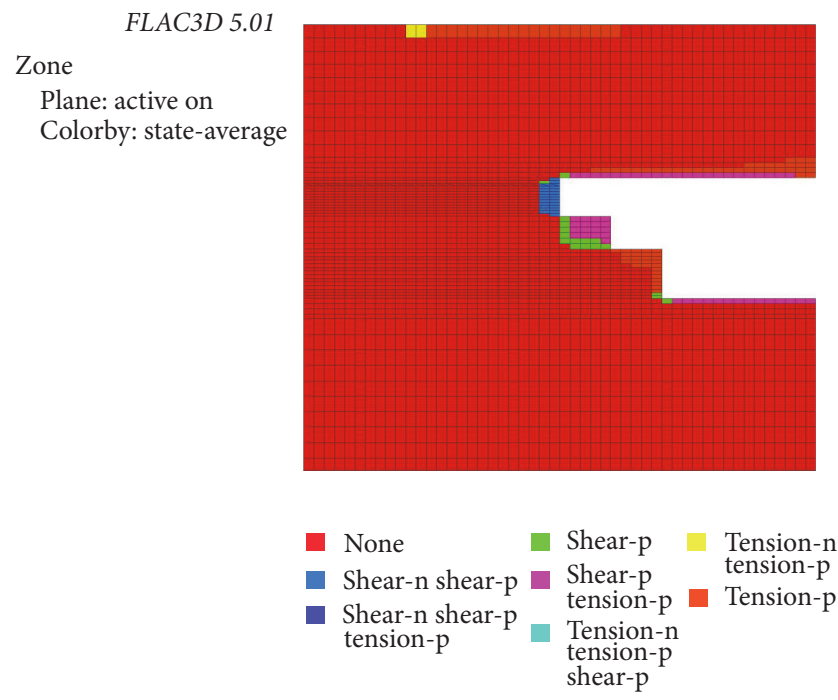

(c) Tribench

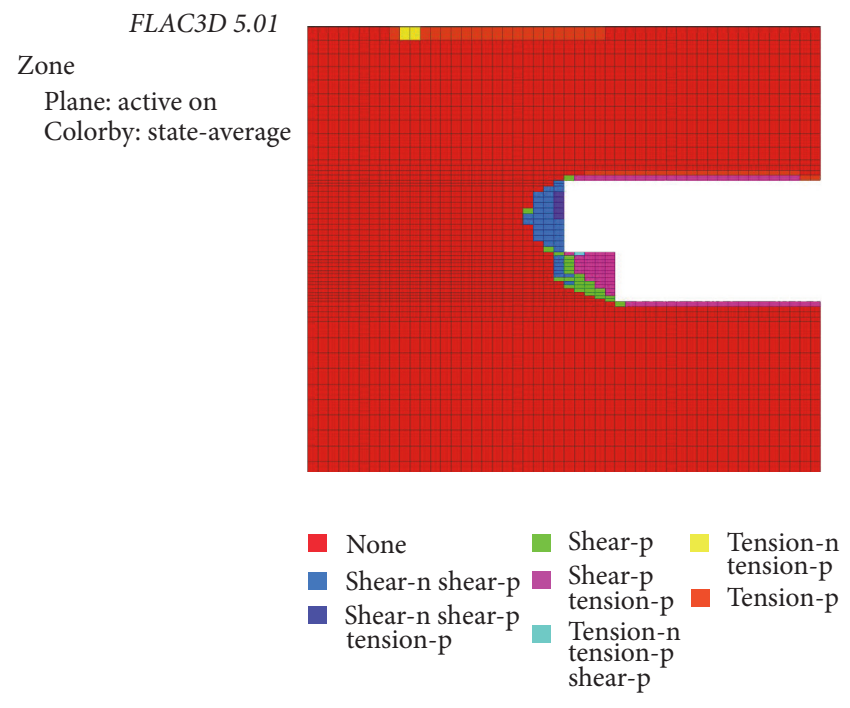

(b) Two-bench section

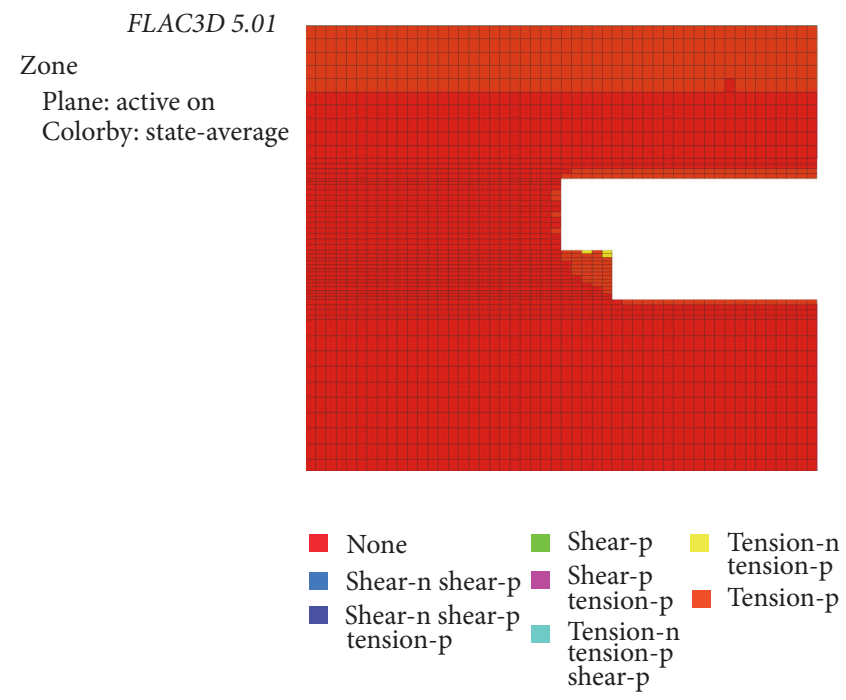

(d) Two-bench section and pregrouting

FIgURE 7: The distribution of the plastic zone deformation.

gradually when excavated to $46 \mathrm{~m}$. For the bench method, the largest settlement due to excavation is still the tribench method.

From Figure 6(d), in the crown settlement curve, in the range of starting excavation to $45 \mathrm{~m}$ footage, the settlement caused by the three methods is not quite different. Moreover, the full-section method tends to stabilize earlier than others, and the cumulative deformation of the two-bench and tribench methods is about 1.6 times that of the full section; also, the deformation amount of the tribench method is still larger than of the two-bench method.

From the deformation results of the clearance convergence and the ground settlement, we found that different methods have different advantages. The analysis of plastic zone failure is required in the further comparison.
4.2.3. The Tunnel Face Displacement and Plastic Zone Failure. For the weak surrounding rock, it is very important to prevent the landslide caused by the tunnel face construction. The tunnel failure mechanism in adverse geological conditions can be depicted graphically by numerical simulation, so as to obtain the potential risks of tunnel construction in different conditions; then, positive prevention has great significance for information construction.

In Figure 7, the numerical simulation compares the displacement of the tunnel face and the development of the plastic zone after excavation in the three methods, analyzing the effect of the small duct grouting to stabilize the tunnel face and confirming the pros and cons of the different conditions on the control of the tunnel face displacement, which provides guidance for tunnel construction. 
TABLE 2: Frequency of convergence displacement measurement.

\begin{tabular}{lccc}
\hline $\begin{array}{l}\text { Displacement speed } \\
(\mathrm{mm} / \mathrm{d})\end{array}$ & Distance from the working face & Frequency & Remarks \\
\hline$>5$ & $(1 \sim 2) \mathrm{B}$ & $1 \sim 4$ times/1 day & $(1)$ B is the tunnel width. \\
$1 \sim 5$ & $(2 \sim 5) \mathrm{B}$ & 1 time/2 days & $(2)$ When the displacement \\
$0.2 \sim 1$ & $5 \mathrm{~B}$ & 1 time/1 week & rate $>5 \mathrm{~mm} / \mathrm{d}$, consider \\
dangerous, alert timely & No monitoring & \\
\hline 0.2 & & &
\end{tabular}

The plastic zone appeared in the rock strata of the tunnel arch waist after excavation; besides, at the bottom of the wall, due to the stress concentration caused by the geometry of the excavation boundary, a plastic zone is presented with a smaller range but a large strain. Generally speaking, the plastic zone of surrounding rock is mainly distributed in the tunnel arch waist, the side wall, and the bottom of the rock stratum.

In the full-section excavation, a closed plastic zone is formed around the tunnel, and the plastic zone's range in front of the tunnel face is large, so invert construction must be timely and reliable, which increases the construction difficulty.

As for the bench methods, we can see that the plastic zone is smaller than the full section; the reason is that the boundary of the plastic zone in front of the tunnel face of the excavation range is concaved to the face, and the plastic zone in the upper and lower benches appears to be arc-shaped through the area. It is inferred that the tribench excavation method has the best effect on controlling the damage of the face and the smallest range of the plastic zone.

Furthermore, forming the deformation of the plastic zone which applied the two-bench excavation and small duct, the range is smaller than other methods shown in Figure $7(d)$, so the advanced small duct grouting to strengthen the surrounding rock can control the destruction of the surrounding rock well.

4.2.4. Reasonable Choice of Construction Methods. Through analyzing the numerical simulation results of different construction programs, the full-section method to control the deformation effect of the surrounding rock is better than the bench excavation, which is more suitable for the stable surrounding rock but needs higher requirements for the supporting time, increasing the difficulty of construction. However, the tribench method has a large disturbance on the formation and surrounding rock, and the difficulty of closing into a ring timely is higher than in the two-bench method. So, the paper held the view that the two-bench method combined with pregrouting is a better way for the actual construction.

\section{Monitor Measurement Feedback}

As the foundation of information construction, monitoring and measurement are the main basis to test the designed parameters, the rationality of the model, and the evaluation of the construction method, whose authenticity is stronger than of the numerical simulation [11].
5.1. Observation of Clearance Convergence. We adopt the LEICA TS09 total station for noncontact measurement and determine the measurement frequency by the surrounding rock level, tunnel section size, and embedded depth. The frequency of convergence displacement measurement was affected by other factors. Table 2 shows the correspondence between measuring frequency and the displacement speed and also the distance from the working face.

After the excavating of the tunnel, the observation piles are embedded in the horizontal wall and the arch waist of the tunnel. The buried depth is $450 \mathrm{~mm}$, and the location of the convergence points around the tunnel is shown in Figure 8. The paper adopted line $2 \#$ as the monitored object.

DK425 + 969 section was selected to monitor the horizontal convergence, as shown in Figure 9; during the 20day monitoring process, the total accumulated amount was $32.51 \mathrm{~mm}$, and the maximum daily convergence was $6.81 \mathrm{~mm}$.

In the initial excavation of the upper bench, the surrounding rock was greatly disturbed due to the construction blasting, making the convergence rate of the deformation increase obviously, and the average daily deformation rate is about $6.61 \mathrm{~mm} / \mathrm{d}$.

With the steel arch, feet-lock bolt, and other initial supports constructed timely, the convergence rate of the surrounding rock decreased gradually, the accumulated deformation reached $73.8 \%$ of the total deformation on the 6 th day, and the average deformation rate dropped below $1 \mathrm{~mm} / \mathrm{d}$.

After the excavation of the lower bench, the deformation increases again, and the average rate over the seven days was $1 \mathrm{~mm} / \mathrm{d}$. At this stage, the initial support should also be constructed timely to close into a ring, which causes the rapid release of the surrounding rock pressure to be relieved to a certain extent, thus reducing the deformation tendency of the convergence.

5.2. Observation of Ground Settlement. Ground settlement measurement refers to the observation from the setting point on the ground, judging the influence on ground settlement by excavating according to the displacement, and the effect on slope due to the excavation by deformation, which can define the stability of the tunnel support structure.

The analysis of the shallow ground settlement measurement in this project combined with DK425 + 985 section and three measuring sections with a pitch of $10 \mathrm{~m}$ is set up in the tunnel portal along the tunnel axis. Start measurement when the distance between the measured section and the working face is $30 \mathrm{~m}$ and stop when the excavation exceeds the measured section and the settlement stabilizes. Figure 10 


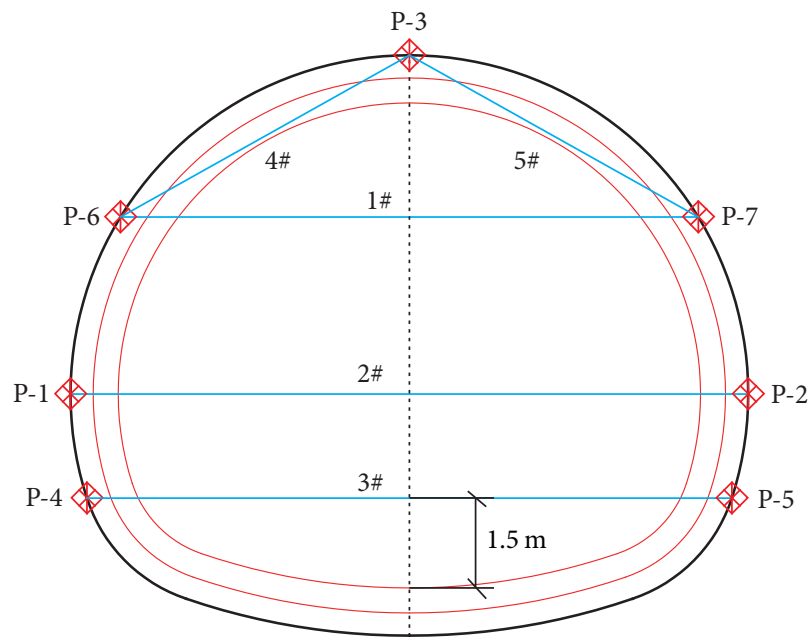

FIGURE 8: Layout of the observation piles.

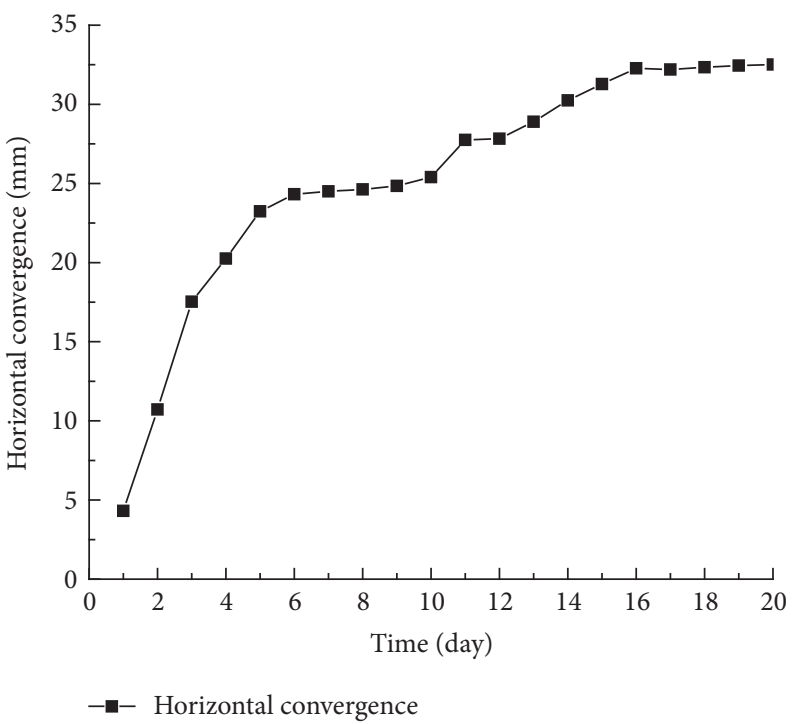

FIGURE 9: Horizontal convergence curve for section DK425 + 969 line 2\#.

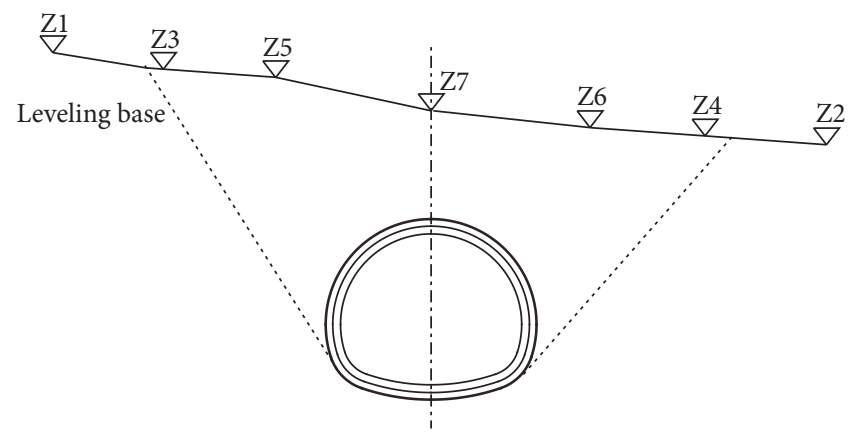

FIGURE 10: The measuring point of entrance and shallow buried section. 
TABLE 3: Frequency of the shallow ground settlement measuring.

\begin{tabular}{llr}
\hline Project & \multicolumn{1}{c}{ Excavation condition } & Measuring frequency \\
\hline \multirow{3}{*}{ The shallow ground settlement } & The distance between excavation and the measured section $<2 \mathrm{~B}$ & $1 \sim 2$ times/d \\
& The distance between excavation and the measured section $<5 \mathrm{~B}$ & 1 time/2 $\mathrm{d}$ \\
& The distance between excavation and the measured section $>5 \mathrm{~B}$ & 1 time/2 weeks \\
\hline
\end{tabular}

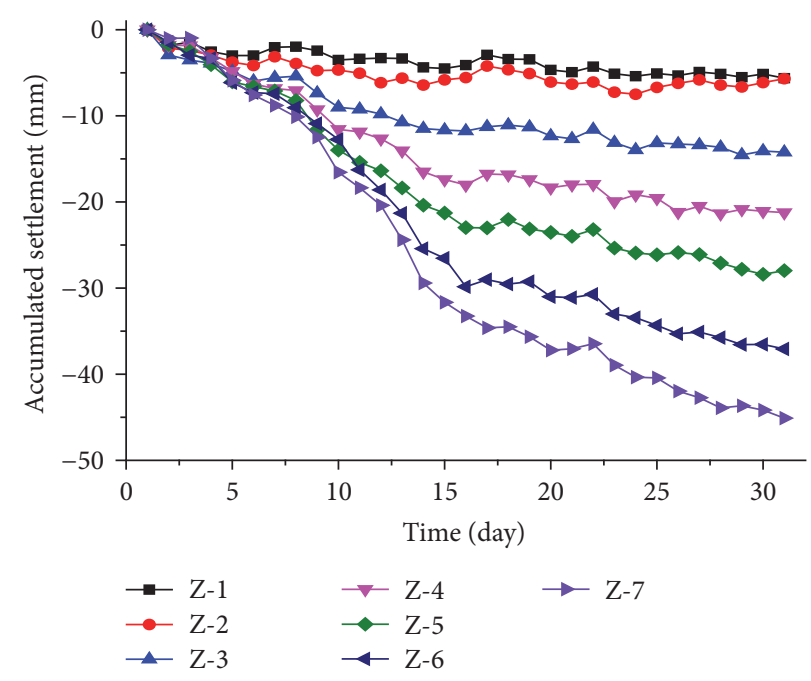

FIGURE 11: Accumulated settlement of the seven measuring points.

shows the location of seven symmetrical measuring points in the entrance of the shallow buried section, and Table 3 shows the measuring frequency.

As shown in Figure 11, we record the accumulated settlement of the seven measuring points, to analyze the deformation process.

Due to the disturbance to the stratum induced by construction in the initial deformation, the original consolidated soil layer became loose, resulting in the bulge or small settlement. The deformation of all the measured points is not obvious, and the smallest is 1 and 2 points with the average deformation of about $7 \mathrm{~mm}$.

As for the bench method, the upper soil is fully excavated, leading to the original balanced three-dimensional soil stress being suddenly destroyed and the geostress being redistributed, causing the deformation of the surrounding soil; moreover, there is the free state of the upper surrounding rock and releasing of a large number of internal stresses. With the initial support that began to play a role, the deformation speed of surrounding rock tends to be gentle. When all the surrounding rock stress is released at the later stage of deformation, the timely construction and backfill of the inverted arch made the rock stable, as well as the ground settlement of each measuring point.

After sixty days of continuous observation, the maximum cumulative deformation of all the measuring points is the 7th point, $45.10 \mathrm{~mm}$, which tends to stabilize. Among all the points, the minimum daily average settling velocity is $0.09 \mathrm{~mm} / \mathrm{d}$, and the maximum is $0.75 \mathrm{~mm} / \mathrm{d}$, which are in the allowable range, indicating that the selected construction method is rational and practical; in addition, the support scheme can effectively control the deformation.

From the information construction point of view, the comparison of numerical model deformation and the monitoring measurement data can be a criterion of reliability in tunnel information model. In addition, the feedback of measurement information is beneficial to the optimization of parameters in the model, and if the relevant parameters are reasonable [12], the numerical model possesses practical reference meaning which can provide more accurate guidance for the subsequent construction.

\section{Conclusions}

In this paper, Xian-nvyan tunnel of Xicheng Railway is taken as the research background, combined with concrete engineering example; an intensive study is carried out by the combination of the geological forecast, numerical simulation, and field monitoring measurement. The main conclusions are as follows.

On the basis of the pregeological investigation, the geological forecast can improve the detailed geological information of the monitoring section, obtain the rock mechanics parameters, and reveal the geological situation in the tunnel area.

By comparing the characteristics of displacement deformation and plastic zone failure in the three construction methods, the deformation mechanism is analyzed. It is concluded that the displacement deformation of the fullsection excavation is smaller than of the bench excavation 
but needs a better geological condition. In contrast, the displacement deformation of the two-bench excavation is in the allowable range and more suitable for the construction of the selected section.

The results of the numerical simulation are the basis of the qualitative analysis, compared with the measured data indicating that the prediction rule is consistent with the actual deformation to some extent, which shows the rationality of the model parameters set by the forecast results.

Only by carrying out numerical simulation and field measuring into design and construction can we ensure the feasibility of mutual certification and feedback and then change the current low level of construction technology quality and apply the concept of information construction to engineering practice.

\section{Conflicts of Interest}

The authors declare that there are no conflicts of interest regarding the publication of this paper.

\section{Acknowledgments}

This research was funded by the National Natural Science Foundation of China (the Experiment and Modeling Studies of the Surface Wavefield by Elliptically Polarization of the Surface Waves) (Approval no. 51508358).

\section{References}

[1] S. Maurhofer, M. Glaettli, and J. Bolliger, "Uetliberg Tunnel: heading methods and interior works," Tunnelling \& Underground Space Technology, vol. 19, p. 349, 2004.

[2] Y.-S. Li, K.-N. Zhang, X. Yang, and C.-B. HuanG, "Numerical simulation for the excavation in tunnel construction," in Proceedings of the International Conference on Civil Engineering and Transportation, pp. 90-93, Trans Tech Publications, Jinan, China, 2011.

[3] Q. Chen, M. Zhao, Y. Zhang, X. Liu, J. Hu, and S. Huang, "Information construction and finite element simulation analysis of unsymmetrical pressure double-arched highway tunnel," Yanshilixue Yu Gongcheng Xuebao/Chinese Journal of Rock Mechanics and Engineering, vol. 25, pp. 1723-1727, 2006.

[4] D. Qiu, S. Li, Y. Xue, and S. Qin, "Prediction study of tunnel collapse risk in advance based on efficacy coefficient method and geological forecast," Journal of Engineering Science and Technology Review, vol. 7, no. 4, pp. 156-162, 2014.

[5] F. Yang, Z.-H. Li, and J.-L. Shi, "Study on application of TSP advanced forecast in tunnel construction," Journal of Railway Engineering Society, vol. 30, pp. 76-80, 2013 (Chinese).

[6] Y. Jia, Y. Li, and H. Liang, "Forecast geological prediction for fault and water gushing in deep-buried super long tunnel," in Proceedings of the Global Conference on Civil, Structural and Environmental Engineering, pp. 1294-1297, Trans Tech Publications, Yichang, China, 2012.

[7] Y. Li, W. Zhu, S. Li, Q. Zhang, H. wang, and W. Kang, "Numerical simulation of a branching-out tunnel construction process using Flac3D," in Proceedings of the 42nd US Rock Mechanics Symposium (USRMS), American Rock Mechanics Association, 2008.
[8] Y. Huang, Z. Wang, Y. Zhao, N. Hao, and Y. Chen, "Numerical simulation of TSP tunnel fault model of seismic prediction," International Journal of Earth Sciences and Engineering, vol. 8, pp. 24-30, 2015.

[9] J. Guo and Q.-C. Wang, "The numerical simulation analysis of excavation process of loess tunnel," in Proceedings of the 2011 International Conference on Manufacturing Science and Technology, (ICMST '11), pp. 6594-6600, Singapore, Singapore, September 2012.

[10] H. B. Fan, J. X. Lai, and D. D. Hou, "The bench method numerical simulation of soft rock tunnel," in Proceedings of the 2nd International Conference on Civil Engineering, Architecture and Sustainable Infrastructure, (ICCEASI '13), Zhengzhou, China, July 2013.

[11] Y. Song, Y. tANG, G. Mu, and G. Wang, "Monitoring of tunnel clearance displacements under condition of TBM construction," Yanshilixue Yu Gongcheng Xuebao/Chinese Journal of Rock Mechanics and Engineering, vol. 28, pp. 621-627, 2009.

[12] J.-H. Yang, J.-Q. Chen, G. He, and Q. Chen, "Research on monitoring and measuring of sandy clay tunnel construction under complex conditions," Yanshilixue Yu Gongcheng Xuebao/Chinese Journal of Rock Mechanics and Engineering, vol. 24, pp. 4588-4593, 2005 (Chinese). 


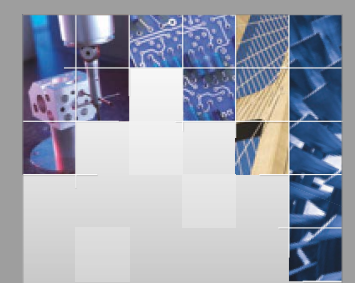

\section{Enfincering}
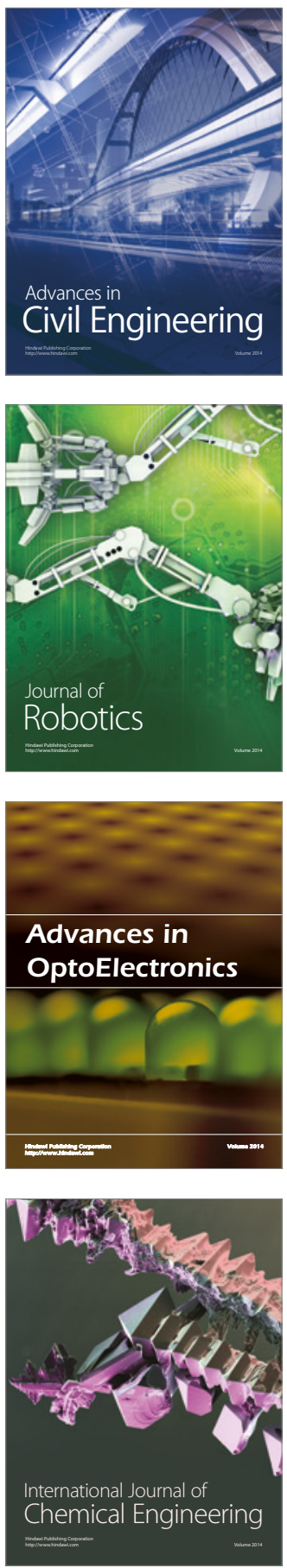

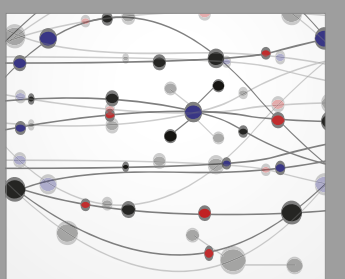

The Scientific World Journal

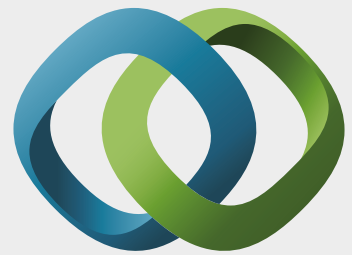

\section{Hindawi}

Submit your manuscripts at

https://www.hindawi.com
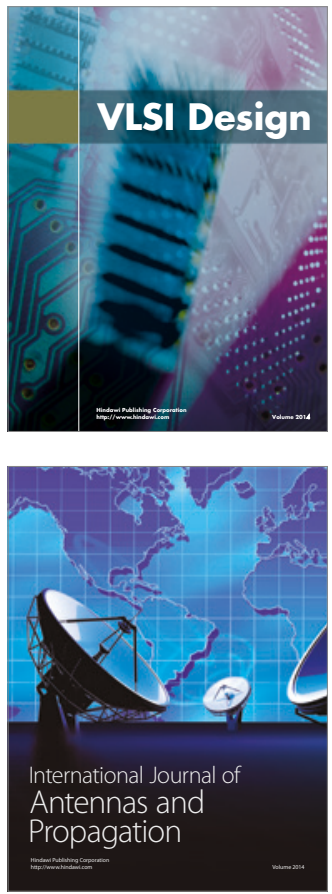

\section{Rotating}

Machinery
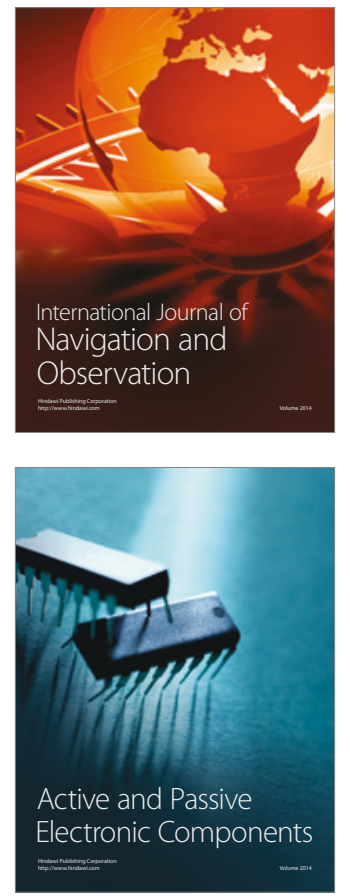
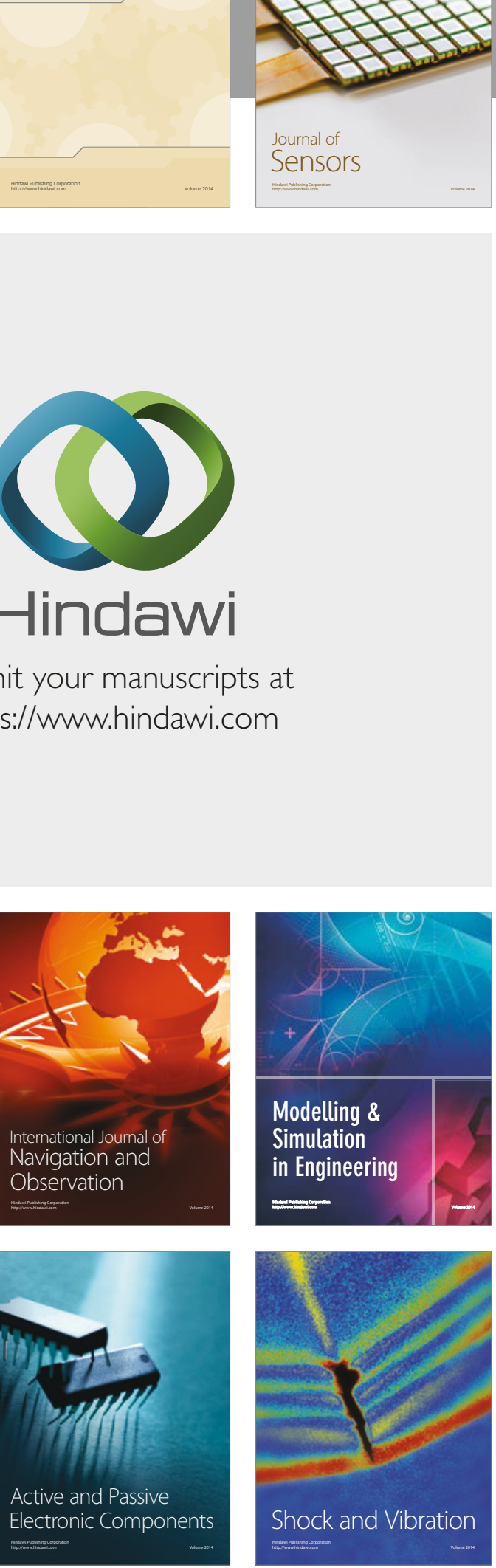
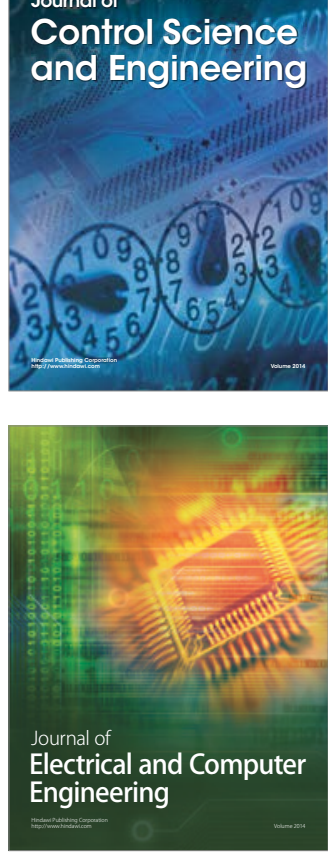

Distributed

Journal of

Control Science

and Engineering
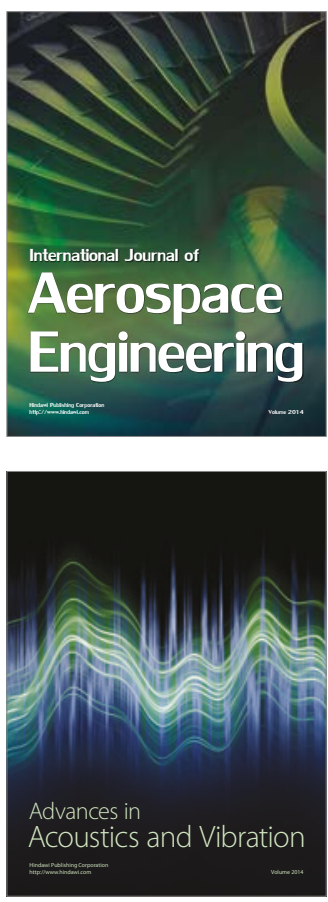

Sensor Networks 